\title{
Effects of letrozole combined with human menopausal gonadotrophin in ovarian stimulation for intrauterine insemination cycles
}

\author{
Xiaoying Yu, ${ }^{1,2 \#}$, Zhiwen $\mathrm{Cao}^{2 \#}$, Wenwen $\mathrm{Hou}^{2}$, Weihua $\mathrm{Hu}^{2}$, Guijun $\mathrm{Yan}^{1}$ \\ ${ }^{1}$ Center of Reproductive Medicine, Nanjing Drum Tower Hospital Clinical College of Nanjing Medical University, Nanjing 210000 , China; ${ }^{2}$ Center \\ of Reproductive Medicine, Yijishan Hospital of Wannan Medical College, Wuhu 241002, China \\ Contributions: (I) Conception and design: G Yan; (II) Administrative support: W Hu; (III) Provision of study materials or patients: W Hou; (IV) \\ Collection and assembly of data: X Yu; (V) Data analysis and interpretation: Z Cao; (VI) Manuscript writing: All authors; (VII) Final approval of \\ manuscript: All authors. \\ \#These authors contributed equally to this work. \\ Correspondence to: Guijun Yan. Nanjing Drum Tower Hospital Clinical College of Nanjing Medical University, Nanjing 210000, China. \\ Email: yanguijun55@163.com
}

\begin{abstract}
Background: The effects of letrozole plus human menopausal gonadotropin (HMG) on ovarian stimulation (OS) of intrauterine insemination (IUI) cycles were examined.

Methods: In all, 1,005 IUI cycles were included in this study. Couples underwent natural cycle (NC) IUI $(n=150)$ or IUI after OS with letrozole $(n=207)$ or IUI after OS with letrozole + HMG $(n=648)$.

Results: The clinical pregnancy rates were $9.0 \%, 13.0 \%$, and $17.0 \%$, and the live birth rates were $7.0 \%$, $9.0 \%$, and $14.0 \%$ in the NC, letrozole, and letrozole + HMG IUI groups, respectively. The twin pregnancy rate of the letrozole + HMG group $(11.0 \%)$ was higher than that of the NC $(7.0 \%)$ and letrozole groups (4.0\%). To date, the only triplet pregnancies to occur were in the letrozole + HMG group. On the day of human chorionic gonadotropin (hCG), the number of follicles with an average follicle diameter greater than $18 \mathrm{~mm}$ in the letrozole $+\mathrm{HMG}$ group $(1.21 \pm 0.56)$ and letrozole group $(1.14 \pm 0.48)$ was greater than that in the NC group $(0.85 \pm 0.36)$. The thickness of the endometrium in the letrozole $+\mathrm{HMG}$ group $(8.8 \pm 2.1 \mathrm{~mm})$ was significantly greater than that in the letrozole group $(7.3 \pm 1.6 \mathrm{~mm})$.

Conclusions: The letrozole + HMG protocol of OS in IUI can improve follicular development, increase the thickness of endometrium, significantly increase the live birth rate, but not significantly increase the multiple pregnancy rate.
\end{abstract}

Keywords: Letrozole; human menopausal gonadotropin (HMG); ovarian stimulation (OS); intrauterine insemination (IUI)

Submitted Nov 02, 2019. Accepted for publication Nov 07, 2019.

doi: $10.21037 /$ atm.2019.11.58

View this article at: http://dx.doi.org/10.21037/atm.2019.11.58

Studies have shown that in many countries, intrauterine insemination (IUI) can be recommended as the first-line treatment for infertile couples who are pursuing treatment for infertility. IUI provides an opportunity to increase the probability of conception, and is simple, fast, and low cost (1). Some studies recommend the use of OS for IUI (2). Other studies have shown that IUI should be performed without
OS (3). A randomized trial showed that after more than 4 IUI cycles, the pregnancy rate for young couples after superovulation was higher $(33 \%)$ than that for unstimulated couples (18\%) (4). However, the clinical pregnancy rate in women aged 40 and above was merely $5 \%$ (5).

A recent retrospective study found that IUI with OS could produce an increase in the upper limit of the 
pregnancy rate of more than $35 \%$ for females and infertile patients with unexplained factors and ovulation disorders. Natural cycle (NC) IUI has its own advantages in improving patients' pregnancy rates with cervical factor infertility, male factor infertility, endometriosis, and other female infertility (6). Huang et al. performed 14,519 IUI cycles in 8,583 couples. Compared with NC IUI (6.2\%), live birth rates were significantly higher in IUI cycles stimulated with the use of clomiphene citrate (CC) (8.9\%), letrozole (9.4\%), and gonadotropins $(9.5 \%)$. The multiple pregnancy rate associated with the $\mathrm{NC}$ was $0.7 \%$, while those associated with the CC cycle, letrozole cycle, and gonadotropin cycle were $4.6 \%, 1.3 \%$, and $4.2 \%$, respectively (7).

Currently, there are many stimulatory drugs in use including CC, gonadotropin-releasing hormone ( $\mathrm{GnRH}$ ) agonists, letrozole, HMG, and combinations of these drugs (8). However, there is no consensus on the best ovarian stimulants. To explore the effectiveness and safety of different OS schemes during IUI treatment for infertile patients and to evaluate the effects of single and multiple follicle growth during IUI treatment, we analyzed the clinical pregnancy rate, live birth rate, and multiple pregnancy rate in natural versus IUI stimulation cycles.

\section{Methods}

\section{Subjects and grouping}

We organized a retrospective study at Yijishan Hospital's Reproductive Medicine Center, Wannan Medical College. All data in this article were retrieved and exported from our clinical database. The research was approved by the local ethics committee and meets ethical demands.

The patients treated with IUI in our study were divided into those treated with or without OS. From January 2014 to December 2018, 658 couples underwent IUI treatment. The inclusion criteria were as follows: (I) couples have normal sexual life without contraception for more than one year without pregnancy, and the wife's hysterosalpingography or laparoscopy results show unilateral or bilateral fallopian tube patency; (II) the husband performed semen analysis with a semen concentration exceeding 5 million per $\mathrm{ml}$ or with the total number of sperm progressive motility greater than 3 million.

The exclusion criteria were as follows: (I) bilateral fallopian tube pathology; (II) ovulation failure after IUI by ultrasound; (III) a total sperm count after semen treatment of $<5$ million per $\mathrm{mL}$ or total number of sperm progressive motility less than 3 million.

\section{NC or OS IUI cycles}

Patients were treated with NC IUI during a regular menstrual cycle. OS was performed in patients with irregular menstruation, abnormal follicle development, or ovulation disorders. Two types of OS protocols (letrozole $v s$. letrozole + HMG) were applied in this study: for the letrozole protocol, letrozole (Hengrui, Jiangsu Province, China) was administered continuously on the $3^{\text {rd }}$ to $5^{\text {th }}$ day after progesterone-induced menstruation or NC menstruation. The dosage was $2.5-5.0 \mathrm{mg} /$ day for 5 days. For the letrozole plus HMG protocol, the dosage of letrozole was $2.5-5.0 \mathrm{mg}$ per day, and letrozole was administered orally for 5 days. From day 3 to day 5, 75 IU HMG (Livzon, Zhuhai, China) was administered. Depending on the ovarian response, HMG was used at different times.

We used transvaginal sonography (TVS) to monitor follicular growth on the $8^{\text {th }}$ to the $10^{\text {th }}$ day of the patient's cycle. Next, we monitored the follicles every 2 days according to the follicle numbers and sizes. When TVS indicated that there were follicles larger than or equal to $18 \mathrm{~mm}$, we induced oocyte excretion by injection of 5,000 10,000 IU human chorionic gonadotropin (hCG) (Livzon, Zhuhai, China). If more than three dominant follicles were present, the couple was advised to cancel the cycle.

\section{Insemination procedure}

Artificial fertilization was scheduled from 36 to 40 hours after hCG injection. After abstinence from sexual activity for 3-7 days, semen was collected in the laboratory, and then washed with density gradient centrifugation and evaluated for count and motility of spermatozoa. The patient was fertilized in the artificial insemination room with a fertilization dose of $0.5 \mathrm{~mL}$. After the operation, the patient lay supine for 30 minutes.

\section{Luteal phase support}

Beginning on the $2^{\text {nd }}$ after insemination, progesterone support comprised $20 \mathrm{mg} / \mathrm{d}$ dydrogesterone (Duphaston, Abbott Biologicals, USA) in all patients. Serum hCG levels were measured on the $14^{\text {th }}$ day after insemination to evaluate the occurrence of pregnancy. Ultrasonography was used 
Table 1 Demographics of patients who underwent NC, letrozole, or letrozole + HMG protocol for IUI cycles

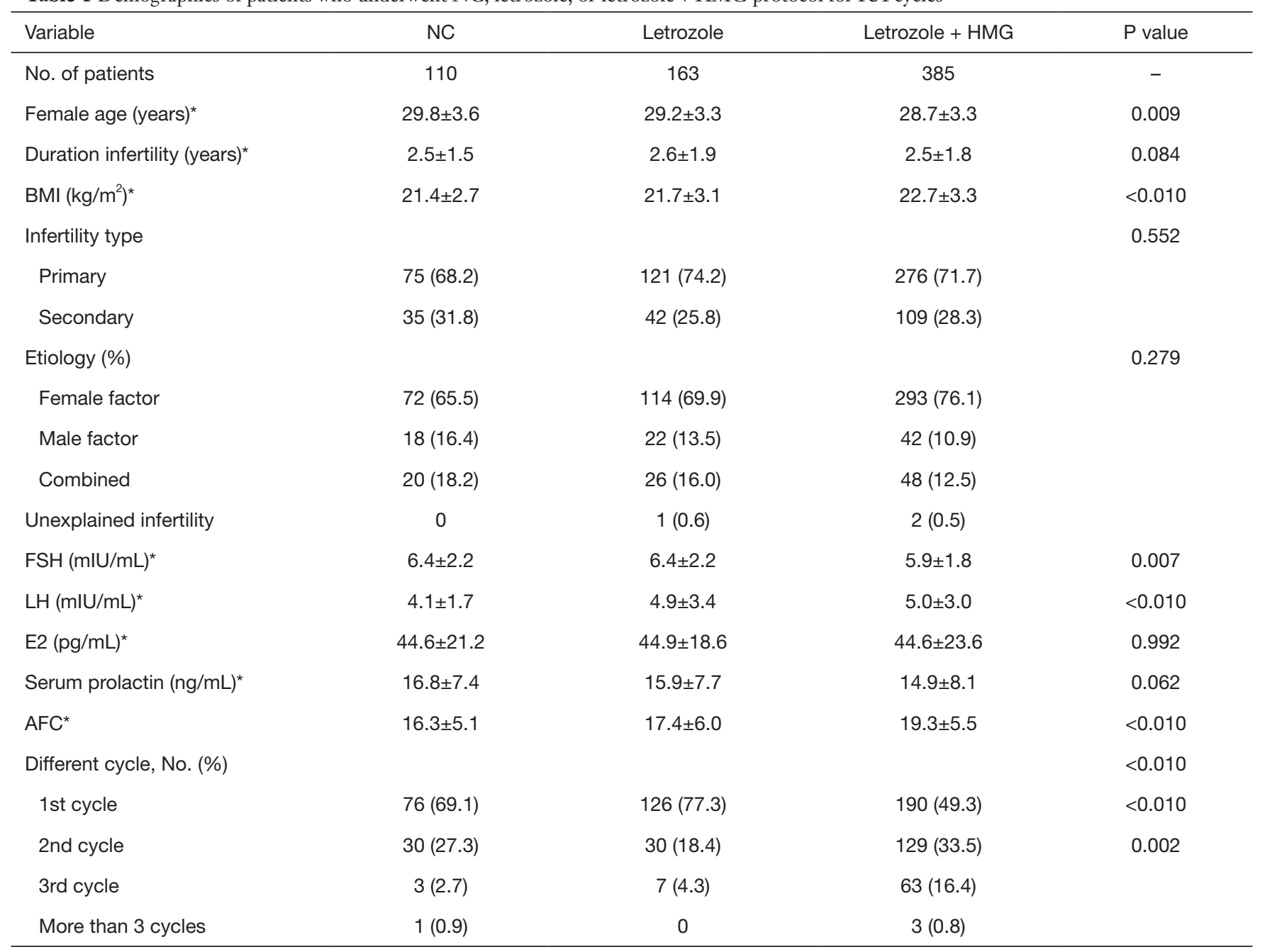

*, results shown are mean SD. NC, natural cycle; HMG, human menopausal gonadotropin; IUI, intrauterine insemination.

16-19 days after artificial fertilization to confirm pregnancy.

\section{Outcomes}

Ultrasonographic findings of an intrauterine pregnancy sac can be was considered clinical pregnancy. Ultrasound examination revealing 2 or more gestational sacs in the uterus indicated multiple pregnancies. Live birth is defined as a fetus born more than 28 weeks pregnant and has signs of normal life after birth.

\section{Statistical analysis}

SPSS18.0 (IBM Corporation, USA) was used for statistical analysis of the data. Measuring data are expressed as mean \pm standard, and counting data are expressed as a ratio. The data were analyzed by variance analysis and a chi-square test. $\mathrm{P}<0.05$ indicated statistical significance.

\section{Results}

From January 2014 to December 2018, data from 658 couples participating in this research were collected. Among the cycles studied, there were $150 \mathrm{NCs}, 207$ letrozole stimulation cycles, and 648 letrozole + HMG cycles. The patients' basic characteristics are displayed in Table 1. The duration of infertility, infertility type, etiology, serum level of estradiol (E2), and PRL were not significantly different between the three groups $(\mathrm{P}>0.05)$. The female age, body mass index (BMI), serum level of follicle-stimulating 
Table 2 A comparison of follicular and endometrial conditions of women undergoing IUI and receiving different protocols of stimulation

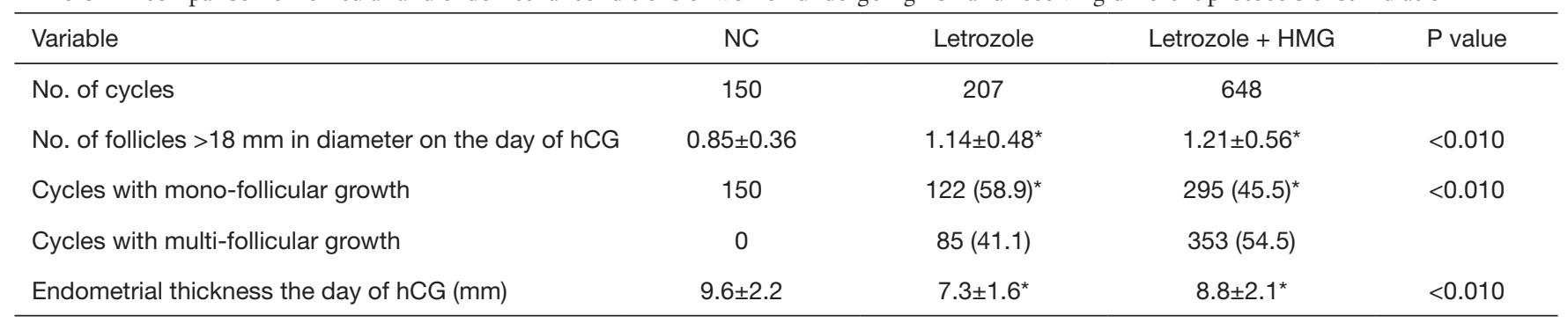

*, $P<0.01$. The $P$ value indicates a statistically significant difference compared with the NC group. NC, natural cycle; HMG, human menopausal gonadotropin; IUI, intrauterine insemination.

Table 3 A comparison of pregnancy outcomes of women undergoing IUI and receiving different protocols of stimulation

\begin{tabular}{|c|c|c|c|c|}
\hline Outcomes & NC & Letrozole & Letrozole + HMG & $P$ value \\
\hline Single pregnancy & $13 / 150$ (0.09) & 25/207 (0.12) & $95 / 648(0.15)$ & 0.128 \\
\hline Twin pregnancy & $1 / 14(0.07)$ & $1 / 26(0.04)$ & $12 / 107$ (0.11) & 0.720 \\
\hline Triplet pregnancy & 0 & 0 & 1 & - \\
\hline Miscarriages & $3 / 14(0.21)$ & $2 / 26(0.08)$ & $15 / 107(0.14)$ & 0.437 \\
\hline Live birth rate & $10 / 150(0.07)$ & 18/207 (0.09) & $88 / 648(0.14)^{*}$ & 0.031 \\
\hline
\end{tabular}

${ }^{*}, \mathrm{P}<0.05$. The $\mathrm{P}$ value indicates a statistically significance difference compared with the NC group. NC, natural cycle; HMG, human menopausal gonadotropin; IUI, intrauterine insemination.

hormone (FSH), serum level of luteinizing hormone (LH), and antral follicle count (AFC) were significantly different between the groups $(\mathrm{P}<0.05)$.

After hCG injection, TVS was used to monitor follicles. The number of follicles with diameters greater than $18 \mathrm{~mm}$ was higher in the letrozole + HMG $(1.21 \pm 0.56)$ and letrozole $(1.14 \pm 0.48)$ groups than that in the $\mathrm{NC}$ group $(0.85 \pm 0.36) \quad(\mathrm{P}<0.010)$. The percentage of women with mono-follicular growth was $100.0 \%, 58.9 \%$, and $45.5 \%$ in NC, letrozole, and letrozole + HMG group, respectively (Table 2). Compared to the letrozole and NC groups, the growth rate for multiple follicles of the cycles in the letrozole + HMG group was higher. Endometrial thickness on the day of hCG injection in the letrozole + HMG group $(8.8 \pm 2.1 \mathrm{~mm})$ was significantly higher than that in the letrozole group $(7.3 \pm 1.6 \mathrm{~mm})$. The NC group's endometrial thickness $(9.6 \pm 2.2 \mathrm{~mm})$ was significantly higher than that of the letrozole and letrozole + HMG groups (Table 2).

Table 3 shows our statistical follow-up. We compared different IUI treatments for OS. The clinical pregnancy rate was $9.0 \%$ after NC, $13.0 \%$ after letrozole, and $17.0 \%$ after letrozole + HMG. Regarding the clinical pregnancy rate, the letrozole group and $\mathrm{NC}$ group had a lower rate than the letrozole + HMG group, but the statistical results showed no significant difference $(\mathrm{P}=0.052)$. In terms of live birth rate, the NC group was the lowest $(7.0 \%)$, and the letrozole + HMG group was the highest (14.0\%). The letrozole + HMG group had the highest live birth rate among the three groups $(\mathrm{P}<0.05)$. Only one triplet pregnancy occurred after treatment with letrozole+HMG.

\section{Discussion}

In the IUI protocols, OS include CC, letrozole, HMG, urinary FSH $(\mathrm{uFSH})$ or recombinant $\mathrm{FSH}(\mathrm{rFSH})$, and $\mathrm{GnRH}$ agonists (9-13). Letrozole is used only for OS or in combination with injectable gonadotropin as an adjuvant (14-16). One of the main advantages of letrozole alone is its ability to restore single follicular ovulation in anovulatory infertility, for example, in polycystic ovary syndrome (PCOS) (17). Franik et al. (9) concluded that compared to 
$\mathrm{CC}$, letrozole could enhance the pregnancy rate and live birth rate of subfertile patients with anovulatory PCOS. OS with letrozole may increase the live birth rate compared with NC IUI without increasing the multiple pregnancy rate in an IUI protocol for unexplained or mild male-factor infertility (7). In ovulating women, letrozole combined with HMG can significantly improve the live birth rate (2). Letrozole can act through different mechanisms in the central and external periphery. Aromatase inhibition improves gonadotropin secretion by decreasing estrogen output from the pituitary and hypothalamus to postmenopausal levels and by transmitting estrogenassociated negative feedback. The peripheral effects of letrozole involve the resulting accumulation of androgens in ovarian follicles when letrozole prevents androgens from converting into estrogens. The accumulation of androgens to a certain extent can promote the expression of the FSH receptor, thereby improving the sensitivity of growth follicles to FSH (9), which may account for the benefits of the combination of HMG and letrozole in OS.

Ovarian-stimulating drugs can stimulate the growth and simultaneous development of multiple follicles in the ovary and endometrium proliferation. A successful pregnancy is affected by implantation, which is a complex process that requires an interaction between the blastocyst and endometrium. Studies have shown that letrozole can activate the $\mathrm{Wnt} / \beta$-catenin pathway, with more endometrial growth in patients with OS using letrozole compared with patients who use CC to stimulate the ovary (18). Letrozole has a good response to the endometrium of PCOS patients throughout the proliferative phase, which is associated with CC (19). The above studies show that letrozole can better increase the thickness of intima compared with CC, which may be the reason for increasing the clinical pregnancy rate.

\section{Conclusions}

In IUI, a protocol with letrozole combined with $\mathrm{HMG}$ for OS can significantly improve follicular development and increase endometrial thickness. Compared with the other two schemes, the letrozole + HMG protocol of OS in IUI can significantly increase the live birth rate, but not significantly increase the multiple pregnancy rate.

\section{Acknowledgments}

Funding: This project was supported by the Science Foundation of Wannan Medical College for Young Scholars
(Grant No. WK2017F28).

\section{Footnote}

Conflicts of Interest: The authors have no conflicts of interest to declare.

Ethical Statement: The authors are accountable for all aspects of the work, and the questions related to the accuracy or integrity of any part of the work are appropriately investigated and resolved. The research was approved by the local ethics committee and meets ethical demands.

\section{References}

1. Group ECW. Intrauterine insemination. Hum Reprod Update 2009;15:265-77.

2. Liu J, Li TC, Wang J, et al. The impact of ovarian stimulation on the outcome of intrauterine insemination treatment: an analysis of 8893 cycles. BJOG 2016;123 Suppl 3:70-5.

3. Goverde AJ, McDonnell J, Vermeiden JP, et al. Intrauterine insemination or in-vitro fertilisation in idiopathic subfertility and male subfertility: a randomised trial and cost-effectiveness analysis. Lancet 2000;355:13-8.

4. Guzick DS, Carson SA, Coutifaris C, et al. Efficacy of superovulation and intrauterine insemination in the treatment of infertility. National Cooperative Reproductive Medicine Network. N Engl J Med 1999;340:177-83.

5. Bou Nemer L, Weitzman VN, Arheart KL, et al. In Vitro Fertilization Versus Mild Stimulation Intrauterine Insemination in Women Aged 40 and Older. Reprod Sci 2017;24:609-12.

6. van Eekelen R, van Geloven N, van Wely M, et al. Is IUI with ovarian stimulation effective in couples with unexplained subfertility? Hum Reprod 2019;34:84-91.

7. Huang $\mathrm{S}$, Wang R, Li R, et al. Ovarian stimulation in infertile women treated with the use of intrauterine insemination: a cohort study from China. Fertil Steril 2018;109:872-8.

8. Haqnawaz F, Virk S, Qadir T, et al. Comparison of Letrozole and Clomiphene Citrate Efficacy along with Gonadotrophins in Controlled Ovarian Hyperstimulation for Intrauterine Insemination Cycles. J Reprod Infertil 2013;14:138-42.

9. Franik S, Eltrop SM, Kremer JA, et al. Aromatase inhibitors (letrozole) for subfertile women with polycystic ovary syndrome. Cochrane Database Syst Rev 
2018;5:CD010287.

10. Moro F, Scarinci E, Palla C, et al. Highly purified hMG versus recombinant FSH plus recombinant $\mathrm{LH}$ in intrauterine insemination cycles in women $>=35$ years: a RCT. Hum Reprod 2015;30:179-85.

11. Huang S, Du X, Wang R, et al. Ovulation induction and intrauterine insemination in infertile women with polycystic ovary syndrome: A comparison of drugs. Eur J Obstet Gynecol Reprod Biol 2018;231:117-21.

12. Demirol A, Gurgan T. Comparison of different gonadotrophin preparations in intrauterine insemination cycles for the treatment of unexplained infertility: a prospective, randomized study. Hum Reprod 2007;22:97-100.

13. Bernacchioni C, Turano P, Donati C. Targeting sphingosine kinase 1 localization as novel target for ovarian cancer therapy. Transl Cancer Res 2017;6:S1277-S1280.

14. Mitwally MF, Casper RF. Use of an aromatase inhibitor for induction of ovulation in patients with an inadequate response to clomiphene citrate. Fertil Steril 2001;75:305-9.

15. Franik S, Kremer JA, Nelen WL, et al. Aromatase

Cite this article as: Yu X, Cao Z, Hou W, Hu W, Yan G. Effects of letrozole combined with human menopausal gonadotrophin in ovarian stimulation for intrauterine insemination cycles. Ann Transl Med 2019;7(23):771. doi: 10.21037/atm.2019.11.58 inhibitors for subfertile women with polycystic ovary syndrome: summary of a Cochrane review. Fertil Steril 2015;103:353-5.

16. Farquhar CM, Liu E, Armstrong S, et al. Intrauterine insemination with ovarian stimulation versus expectant management for unexplained infertility (TUI): a pragmatic, open-label, randomised, controlled, two-centre trial. Lancet 2018;391:441-50.

17. Amer SA, Smith J, Mahran A, et al. Double-blind randomized controlled trial of letrozole versus clomiphene citrate in subfertile women with polycystic ovarian syndrome. Hum Reprod 2017;32:1631-8.

18. Mehdinejadiani S, Amidi F, Mehdizadeh M, et al. Effects of letrozole and clomiphene citrate on Wnt signaling pathway in endometrium of polycystic ovarian syndrome and healthy womendagger. Biol Reprod 2019;100:641-8.

19. Mehdinejadiani S, Amidi F, Mehdizadeh M, et al. The effects of letrozole and clomiphene citrate on ligands expression of $\mathrm{Wnt} 3, \mathrm{Wnt} 7 \mathrm{a}$, and $\mathrm{Wnt} 8 \mathrm{~b}$ in proliferative endometrium of women with Polycystic ovarian syndrome. Gynecol Endocrinol 2018;34:775-80. 